\title{
Sequence requirements for efficient translational frameshifting in the Escherichia coli dnaX gene and the role of an unstable interaction between tRNA ${ }^{\text {Lys }}$ and an AAG lysine codon
}

\author{
Zenta Tsuchihashi and Patrick O. Brown \\ Howard Hughes Medical Institute, and Departments of Pediatrics and Biochemistry, Stanford University Medical Center, \\ Stanford, California 94305 USA
}

\begin{abstract}
Synthesis of the $\gamma$-subunit of DNA polymerase III holoenzyme depends on precise and efficient translational frameshifting to the -1 frame at a specific site in the dnaX gene of Escherichia coli. In vitro mutagenesis of this frameshift site demonstrated the importance of an A AAA AAG heptanucleotide sequence, which allows two adjacent tRNAs to retain a stable interaction with mRNA after they slip to the -1 position. The AAG lysine codon present in the $3^{\prime}$ half of this heptanucleotide was a key element for highly efficient frameshifting. A tRNA ${ }^{\text {Lys }}$ with a CUU anticodon, which has a strong affinity for AAG lysine codons, is present in eukaryotic cells but absent in $E$. coli. Expression in $E$. coli of a mutant tRNA ${ }^{\text {Lys }}$ with a CUU anticodon specifically inhibited the frameshifting at the AAG codon, suggesting that the absence of this tRNA in $E$. coli contributes to the efficiency of the $d n a X$ frameshift.
\end{abstract}

[Key Words: dnaX gene; translational frameshifting; in vitro mutagenesis; tRNA ${ }^{\text {Lys }}$ ]

Received November 14, 1991; revised version accepted January 8, 1992.

In Escherichia coli, a highly efficient translational frameshift is used to produce the $\gamma$-subunit of DNA polymerase III holoenzyme. This frameshift leads to termination at a stop codon in the -1 frame of the $d$ naX gene and is so efficient that the amount of $\gamma$-subunit produced is comparable to that of a full-length in-frame product, r-subunit (Blinkowa and Walker 1990; Flower and McHenry 1990; Tsuchihashi and Kornberg 1990).

The mRNA region required for this frameshift consists of two parts. One is a putative stem-loop structure located downstream of the frameshift site, which may cause the translational machinery to pause (Tsuchihashi 1991). The other is the nucleotide sequence around the frameshift site. These two features were first identified in the translational frameshift site of eukaryotic viruses (Jacks et al. 1988b). In the Rous sarcoma virus (RSV) gag/pol frameshift site, the critical sequence is an A AAU UUA heptanucleotide, where the frameshift takes place from the UUA leucine codon. The simultaneous slippage model proposed by Jacks and Varmus offered an explanation for the role of this heptanucleotide sequence (Jacks et al. 1988b). In this model two adjacent tRNAs, presumably aminoacyl-tRNA and peptidyltRNA, are required to slip back to the -1 frame simultaneously. Although it is possible that this slip happens during or after the peptidyl transfer so that the tRNAs that slip are not aminoacyl-tRNA and peptidyl-tRNA, for convenience we refer to these two tRNAs as A-tRNA and P-tRNA, respectively. In RSV the P-tRNA appears to slip from AAU to AAA, and the A-tRNA from UUA to UUU. According to the simultaneous slippage model, both tRNAs maintain stable interactions with codons after the slip because the first and second nucleotides of the codon are the same in the 0 frame and the -1 frame. The heptanucleotide sequences that allow these interactions can be generalized as X XXY YYZ. Frameshifting in other animal viruses, including the human immunodeficiency virus (HIV) (Jacks et al. 1988a; Wilson et al. 1988 ) and the avian infectious bronchitis virus (IBV) (Brierley et al. 1989), also involves heptamers of this kind. Frameshifting can occur by a similar mechanism in $E$. coli (Sekine and Ohtsubo 1989; Weiss et al. 1989). The dnaX frameshift site has an A AAA AAG sequence that satisfies the XXXY YYZ rule (Blinkowa and Walker 1990; Flower and McHenry 1990; Tsuchihashi and Kornberg 1990). Mutations in this heptanucleotide sequence have been shown to inhibit the frameshift (Tsuchihashi and Kornberg 1990), but a systematic analysis of the effects of nucleotide changes in this heptamer has not been performed previously in this context. 
In this paper we describe an investigation of the codon-anticodon interaction at the shifty heptanucleotide motif in the dnaX gene. Our results verify the importance of favorable codon-anticodon pairing at two adjacent codons in the -1 frame, and point to a weak interaction between $E$. coli tRNA $^{\text {Lys }}$ and an AAG lysine codon as a key determinant of efficient frameshifting in this system.

\section{Results}

Importance of the $A A A A A G$ sequence for frameshifting

The mRNA sequence around the frameshift site of dnaX is shown in Figure 1. The underlined A AAA AAG sequence, which satisfies the X XXX YYZ rule for simultaneous slippage (Jacks et al. 1988b), has been shown by protein sequence analysis to be the site of a -1 frameshift. A putative downstream stem-loop structure enhances this frameshifting (Tsuchihashi and Kornberg 1990).

To investigate the role of this heptameric sequence in dnaX, we examined a series of point mutations in the heptamer region for their effects on frameshifting. $\mathrm{Mu}$ tated genes were expressed using an inducible T7 RNA polymerase gene. After induction by isopropyl $\beta$-thiogalactopyranoside (IPTG), amounts of $\tau$-subunit (the inframe product) and $\gamma$-subunit (the frameshift product) were determined by Western blotting. The frameshift efficiency was calculated from the amounts of the two polypeptides. Figure 2A shows the results of this analysis. Any change introduced into the heptamer decreased frameshifting, whereas mutations outside the heptamer had little or no effect. The whole A AAA AAG sequence is therefore important for efficient frameshifting. $\mathrm{Mu}$ tant ins l (Fig. 2B), which has a 3-nucleotide insertion between the heptanucleotide sequence and the putative

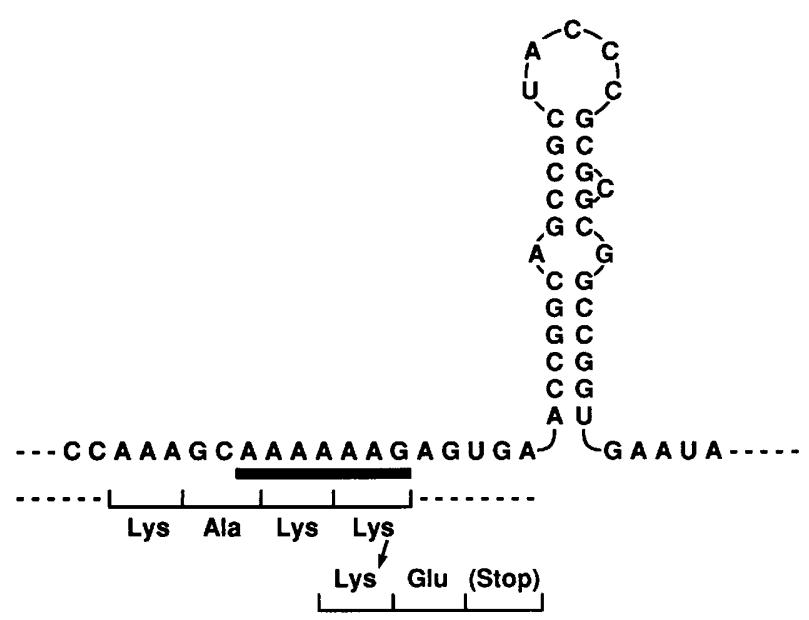

Figure 1. Proposed mRNA structure around the frameshift site in the dnaX gene. stem-loop, still showed efficient frameshifting, although not as efficient as wild type. Thus, there is some flexibility in the required spacing between the dnaX frameshift site and the predicted stem-loop structure, in contrast to the IBV frameshift site, where the precise spacing from the downstream pseudoknot structure is critical (Brierley et al. 1989).

Mutant 4G, which contains an A AAG AAG heptamer (Fig. 2B), retains surprisingly efficient frameshifting $(46 \%)$. Simultaneous slippage on this heptanucleotide would require a codon-anticodon interaction with a G-U base pair at the first position of the triplet, which is never observed in standard codon decoding. This result, therefore, raised the possibility that slippage need not be simultaneous but can involve the upstream A AAG alone. Introduction of an additional mutation in the downstream AAG codon inhibited frameshifting (Fig. 2B; 4G6U, 4G7C, or 4G7A), however, suggesting that frameshifting still involves tRNA slippage at this codon. Perhaps the interaction between tRNA ${ }^{\text {Lys }}$ and the GAA codon in the -1 frame is stabilized to some extent by the G-U base pair at the first position of this codon. The absence of detectable frameshifting in mutant 1G4G (G AAG AAG), however, suggests that simultaneous slippage from AAG to GAA at both sites is very inefficient. Alternatively, the change of the AAA codon to an AAG codon might enhance the slippage of the P-tRNA at this position, partially compensating for the unfavorable UUU/GAA pairing in the -1 frame. As we discuss later, slippage does occur more readily from an AAG codon than from an AAA codon.

The results in Figure 2, suggesting that frameshifting in $d n a X$ occurs by a simultaneous slippage of two adjacent tRNAs, led us to examine whether other sequences that satisfy the X XXY YYZ rule can support frameshifting. Single $A \rightarrow G$ changes at position 1, 2, or 3 (first 3 nucleotides of the heptamer) reduced the frameshift frequency by at least fourfold (Fig. 2A), but when all of these positions were simultaneously changed to $G$, efficient frameshifting was restored (Fig. 2B; mutant 123G). Not only $3 \mathrm{Gs}$, but also 3 Cs or 3 Us in these positions supported efficient frameshifting, although none of them was as favorable as the original 3 As (Fig. 2B; mutants $123 \mathrm{C}$ and $123 \mathrm{U} /$. Thus, the identity of the first 3 nucleotides in the heptamer, which should provide the codon to which the P-tRNA slips, is of secondary importance as long as they are identical.

The sequence requirements for positions 4-7, which provide the site for the A-tRNA to slip, are different. We compared the mutant heptameric sequence A AAG GGA (456G7A) with G GGA AAG (123G) and A AAG AAG (4G) (Fig. 2B,C). It is clear that 456G7A does not allow efficient frameshift but $123 \mathrm{G}$ and $4 \mathrm{G}$ do. From this result, we conclude that the sequence G GGA promotes frameshifting only when it is the site for the P-tRNA slippage, whereas A AAG can promote slippage of either A-tRNA or P-tRNA. The requirements for the slippage of the A-tRNA are thus different and, perhaps, more strict than those for the P-tRNA.

A striking implication of these results is that an AAG 
A

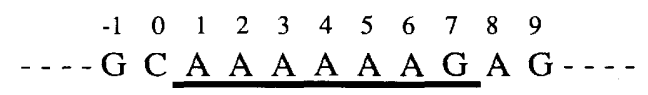

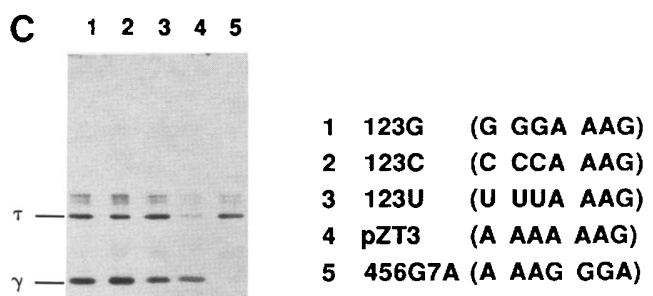

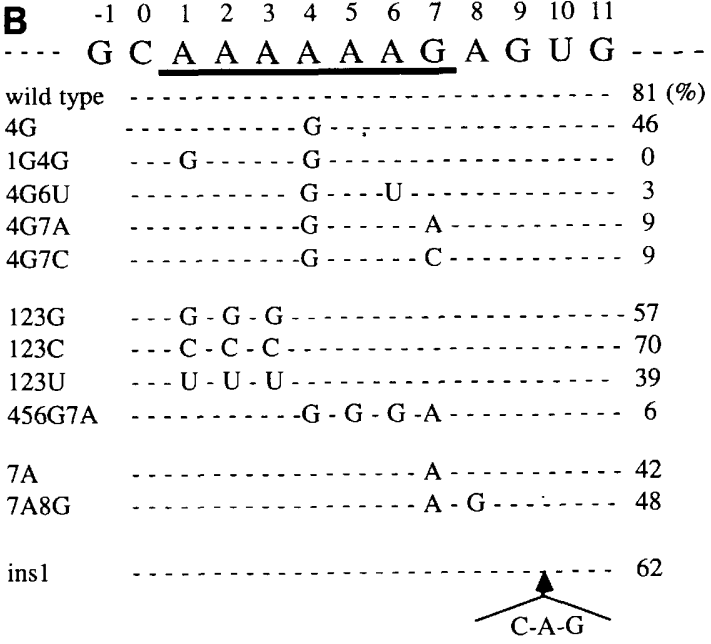

Figure 2. $(A, B)$ Frameshift frequency (percent) for mutants with point mutations. Point mutations were introduced in the sequence around the heptamer motif of the pZT3 dnaX overproducing plasmid (Tsuchihashi and Kornberg 1989). Mutated plasmids were introduced into $E$. coli BL21(DE3) cells, and the amounts of $\tau$ and $\gamma$ were determined by Western blotting after IPTG induction. The molecular ratio of $\tau$ and $\gamma$ was determined, and frameshift frequency was calculated as the number of $\gamma$ per the number of $\tau$ plus $\gamma(\times 100 \%)$. The wild-type gene (pZT3) had a frameshift frequency of $81 \%$ in this experiment. (C) Expression of $\tau$ and $\gamma$ from wild-type (A AAA AAG) and 123G (G GGA AAG), 123C (C CCA AAG), 123U (U UUA AAG), and 456G7A (A AAG GGA) mutants. Calculated frameshift efficiencies are shown in $B$. $\left[\left(^{*}\right)\right.$ This frequency is artificially high owing to transcriptional frameshifting by T7 RNA polymerase.]

codon is sufficient to promote substantial frameshifting in a large variety of sequence contexts. The in vivo consequences of such promiscuous shiftiness might be mitigated if frameshifting at suboptimal heptamers had a greater dependence on the enhancing effect of the putative downstream stem-loop structure. We therefore compared the effect of a disruption in the putative stemloop on frameshifting at a mutant and a wild-type heptamer. A destabilizing change of nucleotide sequence in the stem-loop region from CCGG to AUCC (see Fig. 1) reduced the frameshifting efficiency similarly for both wild-type (from 84-42\%) and mutant 123G (from 63$23 \%$ ) frameshift sites in this experiment (data not shown). It therefore appears possible that diverse heptamer sequences terminating in A AAG are able to promote substantial translational frameshifting even in the absence of a downstream stem-loop structure. How this effect is avoided, tolerated, or exploited in vivo remains an unanswered question.

\section{Slippage of T7 RNA polymerase on the A stretch}

Mutant 7A, which has an A AAA AAA heptanucleotide, gave fairly efficient production of $\gamma$-subunit in an in vivo assay system, which depends on T7 RNA polymerase (Fig. 2A). In vitro transcription-translation of this mutant with an $E$. coli S-30 system supplied with T7 RNA polymerase and rifampicin produced $\gamma$ even more efficiently than the wild-type sequence (Fig. 3A). Surpris- ingly, when the gene was transcribed instead by $E$. coli RNA polymerase, very little $\gamma$ was produced by this mutant, although $\gamma$ was still efficiently formed from the wild-type gene under the same conditions (Fig. 3B). Thus, efficient production of $\gamma$-subunit from mutant 7A depended on transcription by $\mathrm{T} 7$ RNA polymerase. Moreover, mRNA transcribed from this mutant gene by T7 RNA polymerase could direct very efficient production of $\gamma$ in a rabbit reticulocyte lysate translation system, although this system does not support frameshifting in the wild-type dnaX gene (Fig. 3C).

The likely explanation for this T7 RNA polymerasedependent production of $\gamma$ is that T7 RNA polymerase slips during transcription on the extended A stretch in mutant 7A, inserting an extra A. It has been shown previously that $E$. coli RNA polymerase can insert an extra A when it transcribes a sequence of 10 or more consecutive As. Yeast RNA polymerase II was not prone to misincorporation when the same template was used (Wagner et al. 1990). Our results suggest that T7 RNA polymerase is prone to insert an extra $A$ at an even shorter A stretch. Because position 8 is also an A, mutant $7 \mathrm{~A}$ has a stretch of $8 \mathrm{As}$. Because mutant 7A8G showed similar $\gamma$ production in our T7 RNA polymerase-dependent expression system (Fig. 3), a stretch of 7 As seems to be sufficient. A stretch of 6 consecutive As, however, did not appear sufficient to promote this slip, because mRNA transcribed by T7 RNA polymerase from the wild type-gene, or mutants $7 \mathrm{C}$ or $7 \mathrm{U}$, failed to direct production of $\gamma$ in a reticulocyte lysate translation system (Fig. 3C). 
Figure 3. Expression of $\tau$ and $\gamma$ in vitro from wild type (A AAA AAG) and mutants 7A (A AAA AAA), 7C (A AAA AAC), and 7U (A AAA AAU). (A) DNA-dependent transcription-translation coupled system with an $E$. coli S-30 extract supplemented with rifampicin and T7 RNA polymerase. $|B|$ DNA-dependent transcription-translation system without rifampicin or T7 RNA polymerase. $(C)$ Translation in rabbit reticulocyte lysate with mRNA synthesized in vitro by T7 RNA polymerase.
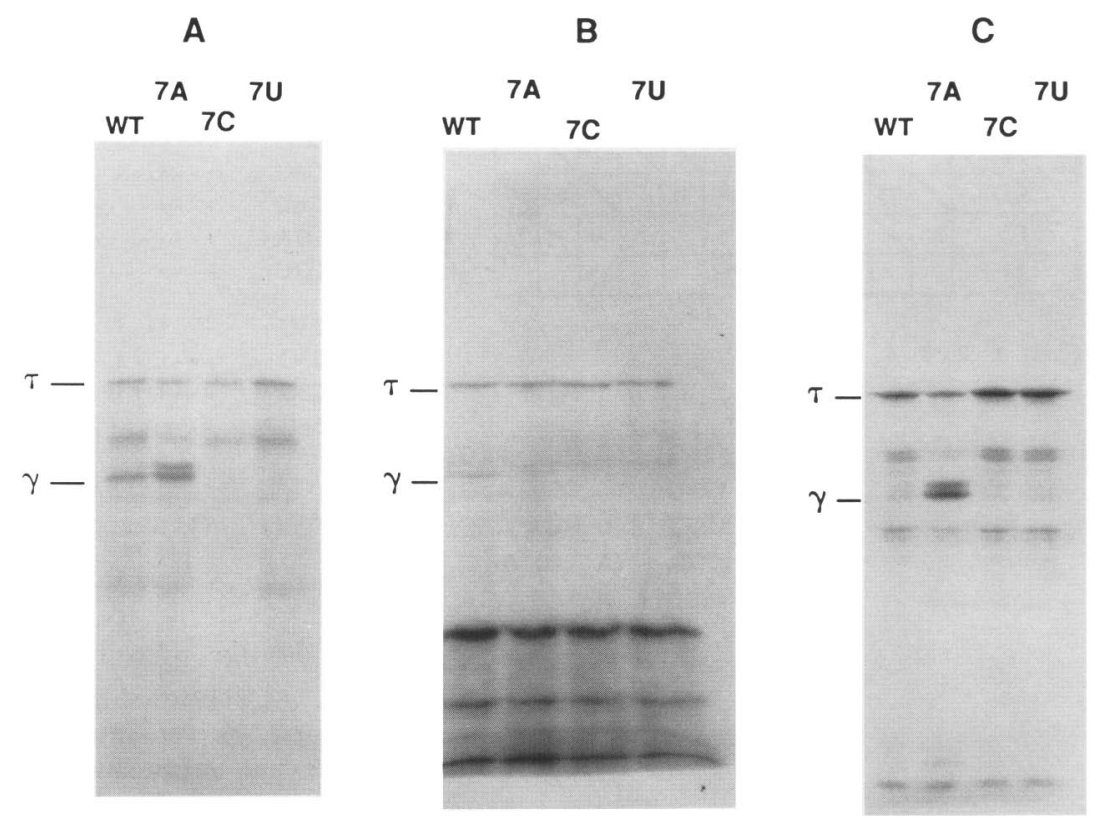

$A A G$ at the second codon is essential for efficient frameshifting

Any nucleotide at position 7 can satisfy the X XXY YYZ rule, but our analysis of mutations at this position (Figs. 2 and $3 A, B)$ showed that a $G$ was required at position 7 for efficient translational frameshifting in E. coli. A similar result was obtained by Weiss and colleagues (1989), studying the expression of wild-type and mutated mouse mammary tumor virus (MMTV) gag/pro frameshift sites in $E$. coli . The A AAA AAC sequence at this MMTV site had to be altered to A AAA AAG to allow vigorous frameshifting in $E$. coli, although the original heptamer promoted efficient frameshifting in a rabbit reticulocyte lysate system (Jacks et al. 1987; Moore et al. 1987; Weiss et al. 1989; Chamorro et al. 1991).

Using a rabbit reticulocyte lysate system, we detected little or no production of $\gamma$ from wild-type (A AAA AAG), mutant 7C (A AAA AAC) or mutant 7U (A AAA AAU) mRNA (Fig. 3C). (The only exception was mutant $7 \mathrm{~A}$, from which $\gamma$ production is presumed to be the consequence of a transcriptional slippage.) The failure of an A AAA AAC sequence in the context of the dnaX gene to direct efficient frameshifting in the mammalian system may be the result of the absence of a pseudoknot structure (Pleij et al. 1985) in the dnaX frameshift region. Such a structure, placed downstream of the shifty heptamer, is required for efficient frameshifting at the A AAA AAC heptamer in MMTV and in several other eukaryotic systems (Brierley et al. 1989; Chamorro et al. 1991; Dinman et al. 1991).

\section{Absence of $t R N A^{\text {Lys }}$ with a CUU anticodon} in $\mathrm{E}$. coli contributes to the efficient frameshifting in the dnaX gene

Why is A AAA AAG such a good frameshift site in $E$. coli, but not in mammalian cells (Fig. 3C; Chamorro et al. 1992) or in yeast cells (Dinman et al. 1991)? We have suggested a model involving the difference of tRNA ${ }^{\text {Lys }}$ species in E. coli and eukaryotic cells (Tsuchihashi 1991). Figure 4 shows the anticodons of $E$. coli and mammalian tRNA ${ }^{\text {Lys }}$ molecules (Spinzl et al. 1989). In E. coli, it is likely that all the tRNA genes have been identified, yet no tRNA ${ }^{\text {Lys }}$ with a CUU anticodon has been found (Komine et al. 1990). Thus, both the AAA and the AAG lysine codons are decoded by a single tRNA ${ }^{\text {Lys }}$, which has a $U^{\star} U U$ anticodon where $U^{*}$ is 5-methylaminomethyl-2-thiouridine. 2-Thiouridine derivatives, found at the wobble position in several tRNA species, have been shown to have a much higher affinity for A than $G$ (Lustig et al. 1981; Yokoyama et al. 1985). The interaction of $E$. coli $\mathrm{tRNA}^{\mathrm{Lys}}$ with the AAG lysine codon may therefore be unusually weak, compared with its interaction with an AAA codon. This would favor the slippage from AAG to AAA that needs to occur at the second codon in the A AAA AAG sequence. Mammals and other eukaryotes have a tRNA ${ }^{\text {Lys }}$ with a CUU anticodon. This tRNA is likely to decode most of the AAG lysine codons, as it has a higher affinity for this codon than does tRNA $^{\text {Lys }}$ with a $U^{\star \star} \mathrm{UU}$ anticodon (Lustig et al. 1981).

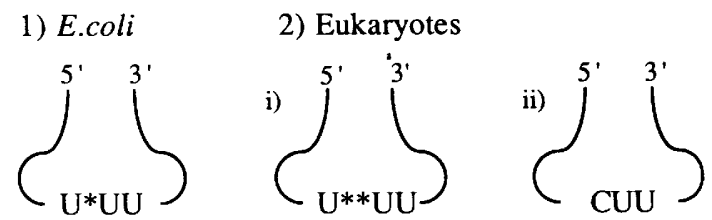

Figure 4. Anticodon structures of tRNA ${ }^{\text {Lys }}$ molecules of E. coli and eukaryotes. $\left(U^{*}\right)$ 5-methylaminomethyl-2-thiouridine; $\left(U^{\star *}\right)$ 5-methoxycarbonylmethyl-2-thiouridine. 
Decoding of an AAG codon by the CUU-type tRNA in eukaryotic cells might resist the slip back.

We tested whether the tRNA ${ }^{\text {Lys }}$ (CUU) could suppress frameshifting at an AAG codon. First, we inserted a DNA fragment containing an E. coli tRNA ${ }^{\text {Lys }}$ gene downstream of the $\operatorname{dnaX}$ gene in plasmid pZT3 so that both tRNA ${ }^{\text {Lys }}$ and the $d n a X$ gene were transcribed by T7 RNA polymerase. $\mathrm{A} \mathrm{T} \rightarrow \mathrm{C}$ change was then introduced in the anticodon region to generate a gene for $\mathrm{tRNA}^{\mathrm{Lys}}$ with a CUU anticodon.

When expression of dnaX was induced along with this novel tRNA, production of $\gamma$ was lower than when the dnaX gene was induced alone (Fig. 5A). The frameshiftsuppressing effect was specific to the mutant tRNA, because overproduction of wild-type tRNA ${ }^{\text {Lys }}$ did not affect the production of $\gamma$ (Fig. 5A). To test whether the effect of the mutant tRNA was specific for the AAG lysine codon, we constructed similar tRNA overproducers coexpressing dnaX genes with mutated frameshift sites (Fig. 5B-D). tRNA ${ }^{\text {Lys }}$ (CUU) inhibited frameshifting at an A AG AAG sequence but had no effect on $\gamma$ production from mutant dnaX genes with either the A AAA AAA or the A AAA AAC sequence. Thus, inhibition of frameshifting by this mutant tRNA clearly depends on the presence of an AAG codon at the frameshift site. The extent of inhibition was far from complete in the experiment shown in Figure $5 \mathrm{~A}$ (from $77-41 \%$ ), probably because there was little mutant tRNA present at the early stage of $d n a X$ induction as a result of the structure of our construct. In fact, when ${ }^{35} \mathrm{~S}$-pulse-labeling was done $1 \mathrm{hr}$ after induction of $d n a X$ and mutant tRNA ${ }^{\text {Lys }}$ expression, inhibition of frameshifting by mutant tRNA was much more pronounced (from $84-19 \%$; Fig. $5 E$ ). Because we do not know how much of the mutant tRNA is transcribed, correctly processed, modified, and charged by lysyl-tRNA synthetase, the observed suppression in this experiment may still be incomplete owning to inefficient production of properly charged tRNA in our system. We conclude that tRNA ${ }^{\text {Lys }}$ with a CUU anticodon can suppress frameshifting on an AAG lysine codon and that the lack of this tRNA in $E$. coli promotes efficient production of the $\gamma$-subunit.

\section{Heptamers related to the dnaX frameshift site in other $\mathrm{E}$. coli genes}

Because A AAA AAG is such an efficient frameshifting signal in $E$. coli, and this sequence can cause a frameshift in the dnaX gene with $6-8 \%$ frequency even without a downstream stem-loop (Tsuchihashi and Kornberg 1990), any E. coli gene with this heptamer in the correct reading frame might be a candidate to express a frameshift product. We searched the GenBank DNA sequence data base for $E$. coli genes in which this motif occurred in the appropriate phase, with an open reading frame of at least 100 codons upstream, and at least 100 codons in

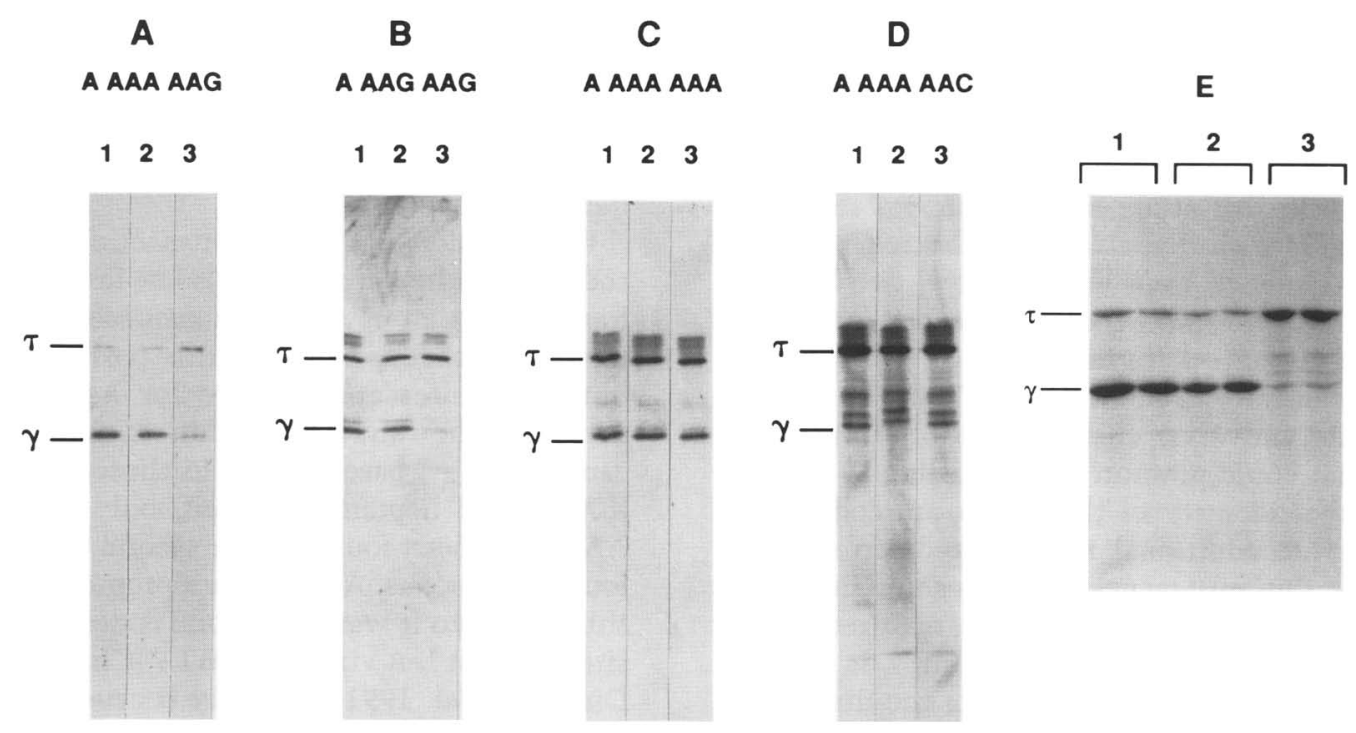

Figure 5. Effect of mutant or wild-type tRNA ${ }^{\mathrm{Lys}}$ on frameshifting. $(A-D) \tau$ and $\gamma$ were visualized by Western blotting. (Lane 1$)$ The expression from the construct without the tRNA ${ }^{\mathrm{Lys}}$ gene; (lane 2) from the construct containing the wild-type tRNA ${ }^{\mathrm{Lys}}$ (U⿱艹一丿) ${ }^{\star} \mathrm{UU}$ anticodon) gene; (lane 3) from the construct containing the mutant tRNA ${ }^{\text {Lys }}$ (CUU anticodon). (A) Effect on wild-type (A AAA AAG) frameshift site. Frameshift efficiency (amount of $\gamma /$ amount of $\tau$ and $\gamma$ ) is calculated as $77 \%$ for no tRNA, 73\% for wild-type tRNA, and $41 \%$ for mutant tRNA. (B) Effect on 4G (A AAG AAG) site. Frameshift efficiency was calculated as $54 \%$ for no tRNA, $65 \%$ for wild-type tRNA, and $17 \%$ for mutant tRNA. (C) Effect on 7A (A AAA AAA) site. Frameshifting efficiency was $34 \%$ for no tRNA, $27 \%$ for wild-type tRNA, and 33\% mutant tRNA. (D) Effect on 7C (A AAA AAC) site. Frameshifting efficiency was $5 \%$ for no tRNA, 7\% for wild-type tRNA, and $9 \%$ for mutant tRNA. $(E)$ Production of $\tau$ and $\gamma$ from the wild-type frameshift site detected by ${ }^{35}$ S-pulselabeling. Frameshifting efficiency determined from the radioactivity in each band and corrected for the number of methionines in $\tau$ and $\gamma$ was $84 \%$ for no tRNA, $85 \%$ for wild-type tRNA, and $19 \%$ for mutant tRNA. 
either the 0 or -1 frame downstream of the heptamer. Eight sequences (the $d n a X, p y r D$, proU, lysR, and ereB genes, open reading frames in the $i l v$ and glycogen gene clusters, and the Ec67 retron) were found to contain the A AAA AAG heptamer in the specified context, two genes $($ fum $A, a s n B)$ contained the A AAG AAG heptamer, and two genes (hly $B$, rhs $A)$ had X XXA AAG heptamers with $\mathrm{X}$ other than A. Except for dnaX, none had a sequence immediately downstream that appeared likely to form a highly stable stem-loop structure. Nevertheless, we are currently investigating the possibility of frameshifting at these sites.

One might expect synonymous alternative sequences to be preferred to potential shifty heptamers, if these sites were prone to translational frameshifting. A search of $E$. coli genes in the GenBank data base for sequences synonymous to the heptamers A AAA AAG, A AAG AAG, or X XXA AAG, appropriately positioned within or between open reading frames, does not reveal such a preference, when the bias in usage of individual synonymous codons is taken into account (Sharp et al. 1988) (data not shown). Moreover, the synonymous nonshifty heptamer A AAG AAA (11 occurrences) is not found significantly more frequently than A AAA AAG (eight occurrences). In the same setting, the first nucleotide is $\mathrm{A}$ in $14 \%$ of N AAA AAG heptamers, versus $13 \%$ of N AAA AAA heptamers, again suggesting that there is little bias against this family of potentially shifty heptamers.

\section{Discussion}

Translational frameshifting in the $d n a X$ gene can be explained by the simultaneous slippage model (Jacks et al. 1988b), which was originally proposed for retroviral frameshifting. In this study we have shown that dnaX frameshifting, as with retroviral frameshifting, requires a heptameric sequence that would allow two adjacent tRNAs to maintain a stable codon-anticodon interaction after slipping to the -1 frame.

Overall conformity to the X XXY YYZ motif was a recurring, but not universal, characteristic of the heptamer sequences that could promote frameshifting in this system. The exact identity of the codon-anticodon pairs involved was an additional important determinant of frameshifting. Our mutagenesis studies show that in the dnaX frameshift signal, the sequence requirement for the second codon, where the A-tRNA slips, is different and apparently more strict than that for the first codon, where the P-tRNA slips. For both codons, it is important that the tRNAs are able to maintain base-pairing at the first and second nucleotide positions after the slip. But for the second codon, this appears not to be sufficientan unstable interaction between tRNA and this codon seems to be an added requirement for efficient frameshifting. In the case of the dnaX gene, it is important that this position is specifically an AAG lysine codon, presumably because of the unstable interaction of this codon and tRNA ${ }^{\mathrm{Lys}}$. One possible explanation for the different requirements at the two sites is that the interaction between P-tRNA and mRNA may be inherently weaker than that between A-tRNA and mRNA. Because the base-pairing between codon and anticodon is not strong enough to explain by itself the accuracy of translation (Hershey 1987), tRNA-mRNA pairing must involve additional interactions mediated by the ribosome or other components. These indirect interactions between P-tRNA and mRNA may be weaker than those between A-tRNA and mRNA, allowing P-tRNA to slip even in the presence of a relatively stable codon-anticodon interaction. A less likely alternative explanation is that slippage of A-tRNA can sometimes occur without slippage of P-tRNA.

The observed preference for the AAG lysine codon in E. coli frameshifting is noteworthy as it is not observed in eukaryotic frameshifting. Recent experiments confirm that the wild-type MMTV frameshift site (A AAA AAC) is a much better frameshift site in eukaryotic systems than a mutant with an A AAA AAG heptamer motif (Chamorro et al. 1991). Our results showed that the heptanucleotide sequence A AAA AAG is especially slippery in $E$. coli because $E$. coli lacks a tRNA ${ }^{\mathrm{Lys}}$ with a CUU anticodon. It has been speculated that translational frameshifting is carried out by specific slippery, or shifty, tRNAs (Weiss 1984; Hatfield et al. 1990). In the case of the frameshift in the yeast Ty1 transposon, a tRNA $^{\text {Leu }}$ with a UAG anticodon, which is specific to the yeast or mitochondrial system, is involved (Belcourt and Farabaugh 1990). Although, unlike retroviral or dnaX frameshifting, this particular frameshift is to the +1 frame and is caused by a slippage of a single tRNA, it is one case where frameshifting depends on the presence of a specific tRNA. Our findings with $d n a X$ show that not only the presence of a specific tRNA, but also the absence of a specific tRNA, can be important for efficient frameshifting.

Weak codon-anticodon interactions at the second codon in the heptamer may also be important for the -1 frameshifts that have been recognized in eukaryotic systems. The codons found at this position in eukaryotic frameshift sites are enriched for As and Us, perhaps avoiding the stronger codon-anticodon interaction caused by G-C base pairing. In those sites that have an X XXY YYZ heptameric motif, only UUA, UUU, and AAC have been found as the second codon. Among the four AAX codons, AAG did not promote efficient frameshifting when it was the second codon in the frameshift sites of yeast L-A virus or MMTV (Chamorro et al. 1991; Dinman et al. 1991), probably because tRNA ${ }^{\text {Lys }}$ with a CUU anticodon is normally present in yeast and mammalian cells. In the L-A viral frameshift site, an AAA codon could induce frameshifting in yeast as efficiently as the original AAC codon. In the present study, efficient transcriptional slippage by T7 RNA polymerase prevented accurate measurement of translational frameshifting at the A AAA AAA heptamer, although it was clearly less efficient than at A AAA AAG (Fig. 3B).

In an MMTV gag/pro context, the A AAA AAA sequence gave little frameshifting in $E$. coli $(\sim 1 / 50$ of that 
dnaX gene frameshifting signal

seen with the A AAA AAG sequence) (Weiss at al. 1989). This species difference is not easily explained by differences in anticodon sequences, because in both cases, the AAA codon is expected to be decoded by a tRNA ${ }^{\text {Lys }}$ with an anticodon of UUU type.

Both AAC and AAU are codons for asparagine. E. coli and mammalian cells usually have only tRNA ${ }^{\text {Asn }}$ with the QUU anticodon, where $\mathrm{Q}$ is a queosine residue, although some cancer cells have tRNA ${ }^{\text {Asn }}$ with the GUU anticodon owing to an incomplete modification (Spinzl et al. 1989). AAC is found more frequently than AAU as the second codon in natural eukaryotic frameshift sites; and when directly compared in the MMTV gag/pro frameshift site, AAC at this position promotes frameshifting at a higher rate than AAU (Chamorro et al. 1991). It seems likely, therefore, that tRNA ${ }^{\text {Asn }}$ (QUU) interacts more strongly with AAU than with AAC.

For UUC and UUU phenylalanine codons, eukaryotes have only tRNA ${ }^{\text {Phe }}$ with a $\mathrm{G}^{\star} \mathrm{AA} / \mathrm{G}^{\star}=2^{\prime}$-O-methylguanosine) anticodon, and in $E$. coli the only tRNA ${ }^{\text {Phe }}$ has a GAA anticodon. These anticodons are expected to interact more strongly with UUC than with UUU. This might account for the observation that UUU is preferred over UUC as the second codon in eukaryotic frameshift sites, and when directly compared, promotes more frequent frameshifting (Jacks et al. 1988b). In E. coli, too, UUU supports more frameshifting than UUC, when tested in the context of the HIV frameshift site (Weiss et al. 1989|. Thus, from the available data on frameshift sites, it appears that the inherent slipperiness of a tRNA can often be predicted from its anticodon sequence. More information on codon-anticodon interactions involving different tRNAs, including unnatural tRNAs modified in vitro (Bruce et al. 1986; O'Connor et al. 1989|, should provide a clearer understanding of the role of specific codons in translational frameshifting.

A search of sequences in the GenBank data base identified several additional $E$. coli genes with an A AAA AAG motif. We do not know yet whether this heptamer promotes frameshifting in any of these genes. In the case of the dnaX gene, the frameshift product is the $\gamma$-subunit of DNA polymerase III holoenzyme, the essential enzyme for DNA replication. Although the $\tau$-subunit is clearly essential for DNA polymerase function, it remains to be determined whether the $\gamma$-subunit is required (O'Donnell 1987; Maki and Kornberg 1988). The demonstration that in vitro, the $\gamma$-subunit complements a mutant allele of $d n a X$ more effectively than does the $\tau$-subunit implies that the $\gamma$-subunit may have an important distinct role in DNA replication (Tsuchihashi and Kornberg 1989). Perhaps this role for the $\gamma$-subunit, and the corollary requirement for efficient translational frameshifting in dnaX, is sufficient to account for the lack of tRNA ${ }^{\text {Lys }}$ (CUU) in E. coli and other prokaryotes. In view of this striking gap in the anticodon table and the resulting propensity for significant frameshifting from AAG codons in diverse sequence contexts, however, the phenomenon of programmed translational frameshifting from an AAG codon may not be confined to the $\operatorname{dn} a X$ gene.

\section{Materials and methods}

DNA construction

Plasmid pZT3 contains the dnaX gene under the control of the T7 phage gene 10 promoter (Tabor and Richardson 1989; Tsuchihashi and Kornberg 1989). All mutations were generated using a polymerase chain reaction (PCR)-mediated mutagenesis method (Helmsley et al. 1989). To construct a plasmid expressing $d n a X$ and tRNA ${ }^{\text {Lys }}$, a DNA fragment containing the $E$. coli tRNA ${ }^{\text {Lys }}$ gene was amplified by PCR from $\lambda p \mathrm{pu}^{+} \beta$ phage clone (Yoshimura et al. 1984) using a pair of PCR primers, 5'-AGC TCT GCA GCG CCG TTG CCA CGG GAT ATC A-3' and 5'-ACG TAT CGA TAC AAC TAA CTA CGC CAG TGG-3', which contain a PstI site and a ClaI site. This fragment was cloned into the PstI and ClaI sites of pZT3 (Tsuchihashi and Kornberg 1989|, both of which are located downstream of dnaX gene.

\section{Quantitation of frameshift efficiency}

All plasmids were introduced into E. coli K12 BL21(DE3) strain (Studier et al. 1990), which contains an IPTG-inducible T7 RNA polymerase gene. Transformed cells were grown in LB medium with $70 \mu \mathrm{g} / \mathrm{ml}$ of carbenicillin at $37^{\circ} \mathrm{C}$, and $1 \mathrm{~mm}$ IPTG was added when the culture reached $\mathrm{OD}_{595}=0.5$. One milliliter of the cell culture was harvested $1 \mathrm{hr}$ after induction, resuspended in $47 \mu \mathrm{l}$ of sample loading buffer (Laemmli 1970), and lysed by boiling. The cell lysates were diluted 1000 -fold and 1-10 $\mu$ l of the diluted sample was subjected to $10 \%$ polyacrylamide gel electrophoresis. After electrophoresis, proteins were transferred to a nitrocellulose filter (Schleicher \& Schuell) by electroblotting, and $\tau$ and $\gamma$ were visualized using rabbit anti- $\gamma$ antiserum and the ECL kit (Amersham). The intensities of the bands were determined with a Molecular Dynamics computing densitometer and compared with those of known quantities of purified $\tau$ and $\gamma$ (Tsuchihashi and Kornberg 1989). For a pulse-labeling experiment, $20 \mu \mathrm{Ci}$ of $\left[{ }^{35} \mathrm{~S}\right]$ methionine (TranS-label, ICN) was added to $0.2 \mathrm{ml}$ of the culture and incubated for $5 \mathrm{~min}$ at $37^{\circ} \mathrm{C}$. The cells were then harvested, and labeled proteins were analyzed by $10 \%$ polyacrylamide gel electrophoresis. Amounts of $\tau$ and $\gamma$ were determined from the radioactivity in each band and corrected for methionine contents. Frameshift efficiency was calculated as (quantity of $\gamma$ /quantity of $\gamma$ plus $\tau) \times 100$.

\section{In vitro translation}

DNA-dependent transcription-translation reactions were carried out using an E. coli S-30 translation kit (Amersham), following the supplier's manual with minor modifications. Ten microliters of the reaction mixture was added to $0.5 \mu \mathrm{g}$ of supercoiled plasmid DNA and $1.5 \mu \mathrm{Ci}$ of $\left[{ }^{35}\right.$ S $]$ methionine (ICN), and was incubated at $30^{\circ} \mathrm{C}$ for $5 \mathrm{~min}$. Cold methionine was added and incubation was continued for an additional $20 \mathrm{~min}$. After the reaction, samples were run on a $10 \%$ polyacrylamide gel (Laemmli 1970) and the gel then was soaked in $1 \mathrm{M}$ sodium salicylate, dried, and subjected to autoradiography. T7 RNA polymerase-dependent reactions were performed under the same conditions except for the addition of $0.4 \mu \mathrm{g}$ of rifampicin and 2 units of T7 RNA polymerase.

The mRNAs for the rabbit reticulocyte lysate translation reactions were synthesized using T7 RNA polymerase and a capping kit (Stratagene), with PstI-digested plasmid DNA template. mRNA $(0.5 \mu \mathrm{g})$ was added to $10 \mu \mathrm{l}$ of rabbit reticulocyte lysate 
(Promega) with $4 \mu \mathrm{Ci}$ of ${ }^{35} \mathrm{~S}$ )methionine and kept at $30^{\circ} \mathrm{C}$ for 45 min. Translation products were detected as described above.

\section{Acknowledgments}

We thank Doug Brutlag for the help in GenBank search, Hachiro Inokuchi for tRNA ${ }^{\text {Lys }}$ clone, Robert B. Weiss, John F. Atkins, and Harold E. Varmus for discussion, and Samson Chow, Melissa A. Parisi, Lee G. Fradkin, Patricia G. Wilson, and Harold E. Varmus for critical reading of this manuscript. This work was supported by National Institutes of Health grant A127205 and by the Howard Hughes Medical Institute (HHMI). P.B. is an assistant investigator of the HHMI.

The publication costs of this article were defrayed in part by payment of page charges. This article must therefore be hereby marked "advertisement" in accordance with 18 USC section 1734 solely to indicate this fact.

\section{References}

Belcourt, M.F. and P.J. Farabaugh. 1990. Ribosomal frameshifting in the yeast transposon Ty1: tRNAs induce slippage on a 7 nucleotide minimal site. Cell 62: 339-352.

Blinkowa, A. and J.R. Walker. 1990. Programmed ribosomal frameshifting generates the Escherichia coli DNA polymerase III $\gamma$-subunit from within the $\tau$-subunit reading frame. Nucleic Acids Res. 18: 1725-1729.

Brierley, I., P. Digard, and S.C. Inglis. 1989. Characterization of an efficient coronavirus ribosomal frameshifting signal: Requirement for an RNA pseudoknot. Cell 57: 537-547.

Bruce, A.G., J.F. Atkins, and R.F. Gesteland. 1986. tRNA anticodon replacement experiments show that ribosomal frameshifting can be caused by doublet decoding. Proc. Natl. Acad. Sci. 83: 5062-5066.

Chamorro, M., N. Parkin, and H.E. Varmus. 1992. An RNA pseudoknot and an optimal heptameric shift site are required for highly efficient ribosomal frameshifting on a retroviral messenger RNA. Proc. Natl. Acad. Sci. 88: 713-717.

Dinman, J.D., T. Icho, and R.B. Wickner. 1991. A - 1 ribosomal frameshift in a double-stranded RNA virus of yeast forms a gag-pol fusion protein. Proc. Natl. Acad. Sci. 88: 174-178.

Flower, A.M. and C.S. McHenry. 1990. The $\gamma$-subunit of DNA polymerase III holoenzyme of Escherichia coli is produced by ribosomal frameshifting. Proc. Natl. Acad. Sci. 87: 37133717.

Hatfield, D., Y.-X. Feng, B.J. Lee, A. Rein, J.G. Levin, and S. Oroszlan. 1990. Chromatographic analysis of the aminoacyltRNAs which are required for translation of codons at and around the ribosomal frameshift sites of HIV, HTLV-1, and BLV. Virology 173: 736-742.

Helmsley, A., N. Arnheim, M.D. Toney, G. Cortopassi, and D.J. Galas. 1989. A simple method for site-directed mutagenesis using the polymerase chain reaction. Nucleic Acids Res. 17: 6545-6551.

Hershey, J.W.B. 1987. Protein synthesis. In Eshcerichia coli and Salmonella typhimurium (ed. F.C. Neidhardt), pp. 613-647. American Society for Microbiology, Washington, D.C.

Jacks, T., K. Townsley, H.E. Varmus, and J. Majors. 1987. Two efficient ribosomal frameshifting events are required for synthesis of mouse mammary tumor virus gag-related polyproteins. Proc. Natl. Acad. Sci. 84: 4298-4302.

Jacks, T., M.D. Power, F.R. Masiarz, P.A. Luciw, P.J. Barr, and H.E. Varmus. 1988a. Characterization of ribosomal frameshifting in HIV-1 gag-pol expression. Nature 331: 280283.

Jacks, T., H.D. Madhami, F.R. Masiarz, and H.E. Varmus. 1988 b.
Signals for ribosomal frameshifting in the Rous sarcoma virus gag-pol region. Cell 55: 447-458.

Komine, Y., T. Adachi, H. Inokuchi, and H. Ozeki. 1990. Genomic organization and physical mapping of the transfer RNA genes in Escherichia coli K12. J. Mol. Biol. 212: 579-598.

Laemmli, U.K. 1970. Cleavage of structural proteins during the assembly of the head of bacteriophage T4. Nature 227: 680685.

Lustig, F., P. Elias, T. Axberg, T. Samuelsson, I. Tittawella, and U. Lagerkvist. 1981. Codon reading and translational error: Reading of the glutamic acid and lysine codons during protein synthesis in vitro. J. Biol. Chem. 256: 2635-2643.

Maki, S. and A. Kornberg. 1988. DNA polymerase III of Escherichia coli: III. Distinctive processive polymerases reconstituted from purified subunits. J. Biol. Chem. 263: 6561-6569.

Moore, R., M. Dixon, R. Smith, G. Peters, and C. Dickson. 1987. Complete nucleotide sequence of a milk-transmitted mouse mammary tumor virus: Two frameshift events are required for translation of gag and pol. J. Virol. 61: 480-490.

O'Connor, M., R.F. Gesteland, and J.F. Atkins. 1989. tRNA hopping: Enhancement by an expanded anticodon. $E M B O H$. 8: $4315-4323$.

O'Donnell, M. 1987. Accessory proteins bind a primed template and mediate rapid cycling of DNA polymerase III holoenzyme from Escherichia coli. I. Biol. Chem. 262: 1655816565.

Pleij, C.W.A., K. Rietveld, and L. Bosch. 1985. A new principle of RNA folding based on pseudoknotting. Nucleic Acids Res. 13: $1717-1731$.

Sekine, Y. and E. Ohtsubo. 1989. Frameshifting is required for production of the transposase encoded by insertion sequence 1. Proc. Natl. Acad. Sci. 86: 4609-4613.

Sharp, P.M., E. Cowe, D.G. Higgins, D.C. Schields, K.H. Wolfe, and F. Wright. 1988. Codon usage patterns in Escherichia coli, Bacillus subtilis, Saccharomyces cerevisiae, Schizosaccharomyces pombe, Drosophila melanogaster, and Homo sapiens; A review of the considerable within-species diversity. Nucleic Acids Res. 16: 8207-8211.

Spinzl, M., T. Hartmann, J. Weber, J. Blank, and R. Zeidler. 1989. Compilation of tRNA sequences and sequences of tRNA genes. Nucleic Acids Res. (Suppl.) 17: 736-742.

Studier, W.F., A.H. Rosenberg, J.J. Dunn, and J.W. Dubendorff. 1990. Use of T7 RNA polymerase to direct the expression of cloned genes. Methods Enzymol. 185: 60-89.

Tabor, S. and C.C. Richardson. 1989. Selective inactivation of the exonuclease activity of bacteriophage T7 DNA polymerase by in vitro mutagenesis. J. Biol. Chem. 264: 6448-6458.

Tsuchihashi, Z. 1991. Translational frameshifting in the Escherichia coli dnaX gene in vitro. Nucleic Acids Res. 19: 24572462.

Tsuchihashi, Z. and A. Kornberg. 1989. ATP interactions of the $\tau$ and $\gamma$-subunits of DNA polymerase III holoenzyme of Escherichia coli. J. Biol. Chem. 264: 17790-17795.

-1990. Translational frameshifting generates the $\gamma$-subunit of DNA polymerase III holoenzyme. Proc. Natl. Acad. Sci. 87: 2516-2520.

Wagner, L.A., R.B. Weiss, R. Driscol, D.M. Dunn, and R.F. Gesteland. 1990. Transcriptional slippage occurs during elongation at runs of adenine or thymine in Escherichia coli. Nucleic Acids Res. 18: 3529-3535.

Weiss, R.B. 1984. Molecular model of ribosome frameshifting. Proc. Natl. Acad. Sci. 81: 5797-5801.

Weiss, R.B., D.M. Dunn, M. Shuh, J.F. Atkins, and R.F. Gesteland. 1989. E. coli ribosomes re-phase on retroviral frameshift signals at rates ranging from 2 to 50 percent. The New Biologist 1: 159-170. 
Wilson, W., M. Braddock, S.E. Adams, P.D. Rathjen, S.M. Kingsman, and A.J. Kingsman. 1988. HIV expression strategies: Ribosomal frameshifting is directed by a short sequence in both mammalian and yeast systems. Cell 55: 1159-1169.

Yokoyama, S., T. Watanabe, K. Murao, H. Ishikura, Z. Yamaizumi, S. Nishimura, and T. Miyazawa. 1985. Molecular mechanism of codon recognition by tRNA species with modified uridine in the first position of the anticodon. Proc. Natl. Acad. Sci. 82: 4905-4909.

Yoshimura, M., M. Kimura, M. Ohno, H. Inokuchi, and H. Ozeki. 1984. Identification of transfer RNA suppressors in $E$. coli: III. Ochre suppressors of lysine tRNA. /. Mol. Biol. 177: 609-625. 


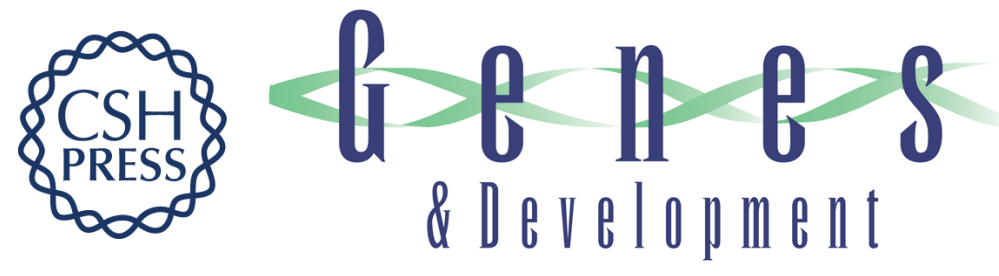

\section{Sequence requirements for efficient translational frameshifting in the Escherichia coli dnaX gene and the role of an unstable interaction between tRNA(Lys) and an AAG lysine codon.}

Z Tsuchihashi and P O Brown

Genes Dev. 1992, 6:

Access the most recent version at doi:10.1101/gad.6.3.511

References This article cites 34 articles, 13 of which can be accessed free at: http://genesdev.cshlp.org/content/6/3/511.full.html\#ref-list-1

License

Email Alerting Service

Receive free email alerts when new articles cite this article - sign up in the box at the top right corner of the article or click here.

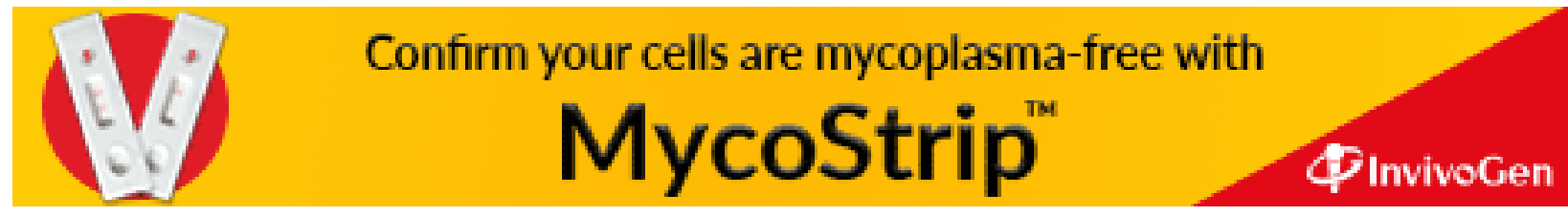

